\title{
Mapping deformed hyperbolic potentials into nondeformed ones
}

\author{
A. de Souza Dutra* \\ UNESP-Campus de Guaratinguetá-DFQ \\ Av. Dr. Ariberto Pereira da Cunha, 333 \\ C.P. 205 \\ 12516-410 Guaratinguetá SP Brasil
}

November 5, 2018

\begin{abstract}
In this work we introduce a mapping between the so called deformed hyperbolic potentials, which are presenting a continuous interest in the last few years, and the corresponding nondeformed ones. As a consequence, we conclude that these deformed potentials do not pertain to a new class of exactly solvable potentials, but to the same one of the corresponding nondeformed ones. Notwithstanding, we can reinterpret this type of deformation as a kind of symmetry of the nondeformed potentials.
\end{abstract}

*E-mail: dutra@feg.unesp.br 
In this work we are interested in analyzing the so called deformed hyperbolic potentials, as introduced by Arai [1] some years ago. We intend to show that this interesting idea should be reinterpreted as a kind of parameter scaling symmetry of the model. The deformed hyperbolic functions introduced by Arai [1], are given by

$$
\sinh _{q}(\alpha x) \equiv \frac{e^{x}-q e^{-x}}{2}, \cosh _{q}(\alpha x) \equiv \frac{e^{x}+q e^{-x}}{2} .
$$

In fact, we show here that by doing a convenient translation of the spatial variable, one can transform the deformed potentials into the corresponding nondeformed ones.

$$
\sinh _{q}(\alpha x)=\sqrt{q} \sinh (\alpha y) ; \cosh _{q}(\alpha x)=\sqrt{q} \cosh (\alpha y)
$$

where

$$
x=y+\frac{1}{\alpha} \ln (\sqrt{q}) .
$$

As examples, we are going to explore some cases of a growing list of models and works devoted to this kind of deformation [1]-[19]. Let us first examine the deformation of the Rosen-Morse potential, used usually to treat molecular interactions, as studied by Eğrifes et al [2], and another one connected to it. They are given respectively by

$$
V_{q}^{I}(x)=B_{0} \tanh _{q}(\alpha x)-U_{0} \sec h_{q}^{2}(\alpha x),
$$

and

$$
V_{q}^{I I}(x)=\frac{V_{1}}{2}\left(1+\tanh _{q}(\alpha x)\right)+\frac{V_{2}}{4}\left(1+\tanh _{q}^{2}(\alpha x)\right) .
$$

In fact, the first of the above potentials was recently considered in a study of the Klein-Gordon equation [19].

It is easy to check that, by performing the above defined translation (3), one recovers the corresponding nondeformed potentials:

$$
V_{q}^{I}(y)=B_{0} \tanh (\alpha y)-\frac{U_{0}}{q} \sec h^{2}(\alpha y),
$$

and

$$
V_{q}^{I I}(y)=\frac{V_{1}}{2}(1+\tanh (\alpha y))+\frac{V_{2}}{4}\left(1+\tanh ^{2}(\alpha y)\right) .
$$


For the first case, we see that the deformed system is nothing but a translation with a corresponding parameter scaling of the well known RosenMorse potential. This shows that there exists a symmetry connecting the Rosen-Morse potential with different coupling parameters. In other words, apart from a shift of the minimum of the potential, the energy spectrum of the potential for different parameters is exactly the same. Regarding the second potential examined in [2], there is even no change of the potential parameters, as can be observed in the figures appearing in that paper.

By starting with the original form of the Rosen-Morse potential [20],

$$
V_{R S}(y)=B \tanh \left(\frac{y}{d}\right)-C \sec h^{2}\left(\frac{y}{d}\right),
$$

and identifying the parameters: $d=\frac{1}{\alpha}$. Then, performing the translation $y=x-\frac{1}{\alpha} \ln (\sqrt{q})$, we obtain the following transformed potential

$$
V_{R S}(x)=B \tanh (\alpha x)-\frac{C}{q} \sec h^{2}(a x) \equiv V_{q}^{I}(y),
$$

so that, in order to complete the connection, we must have that

$$
B \equiv B_{0}, C \equiv U_{0}
$$

In other words, we just need to change $B$ by $B_{0}, C$ by $\frac{U_{0}}{q}$ in the expression of the energy obtained in [20]. So we obtain

$$
E_{n}=-\frac{1}{4}\left[\left(4 \frac{U_{0}}{q}+g^{2}\right)^{\frac{1}{2}}-g(2 n+1)\right]^{2}+\frac{B_{0}^{2}}{\left[\left(4 \frac{U_{0}}{q}+g^{2}\right)^{\frac{1}{2}}-g(2 n+1)\right]^{2}},
$$

where $g \equiv \frac{\hbar^{2} \alpha^{2}}{2 M}$. From above, we can see that changing the potential parameter $U_{0}$ and correspondingly choosing a convenient modification of the translation parameter $q$, we recover the same energy spectrum.

On the other hand, the corresponding eigenfunctions become

$$
\begin{aligned}
\psi_{n}(x)= & N_{n} e^{-a \alpha x} \cosh ^{-b}(\alpha x) \times \\
& \times F\left(-n,(4 \gamma+1)^{\frac{1}{2}}-n ; a+b+1 ; \frac{1}{2}[1+\tanh (\alpha x)]\right),
\end{aligned}
$$


where $N_{n}$ is the normalization constant which we will not specify, once it is not necessary for the purpose of the present work. Furthermore we have defined,

$$
a \equiv-g^{-2} \frac{B}{\left[(4 \gamma+1)^{\frac{1}{2}}-2 n-1\right]} ; b \equiv\left(\gamma+\frac{1}{4}\right)^{\frac{1}{2}}-n-\frac{1}{2}
$$

and $\gamma \equiv g^{-2} C$. Now, by doing our convenient translation we recover:

$$
\begin{aligned}
\psi_{n}(y)= & N_{n} q^{\frac{a-b}{2}} e^{-a \alpha y} \cosh ^{-b}(\alpha y) \times \\
& \times F\left(-n,(4 \gamma+1)^{\frac{1}{2}}-n ; a+b+1 ; \frac{1}{2}[1+\tanh (\alpha y)]\right) .
\end{aligned}
$$

For the other hand, we can also discuss some other cases of the increasing list of works discussing such kind of deformation. For instance, we can cite two recent works [12], [16], which treat a five-parameter exponential-type potential model, both in nonrelativistic [12] as in the relativistic Klein-Gordon case [16]. In particular, they deal with a superpotential with the following basic form

$$
W\left(x, Q_{1}, Q_{2}, Q_{3}\right) \equiv Q_{1}+\frac{Q_{2}}{e^{2 \alpha x}+q}+\frac{Q_{3}}{e^{2 \alpha x}+q} e^{\alpha x},
$$

which, after performing the translation (3) suggested above in the text, one ends with

$$
W\left(x, Q_{1}, Q_{2}, Q_{3}\right) \equiv Q_{1}+\frac{\left(\frac{Q_{2}}{q}\right)}{e^{2 \alpha x}+1}+\frac{\left(\frac{Q_{3}}{\sqrt{q}}\right)}{e^{2 \alpha x}+1} e^{\alpha x},
$$

where one can easily see that a scaling of the parameters eliminates the dependence on the parameter $q$. So, we conclude that there are only four independent parameters after the translation. Finally, let us discuss the so called generalized Morse potential [11], given by

$$
V(x)=V_{1} e^{-2 \alpha x}-V_{2} e^{-\alpha x} .
$$

In this case we perform the following translation

$$
x=y+\frac{1}{\alpha} \ln \left(\frac{V_{1}}{V_{2}}\right),
$$


which leads to

$$
V(x)=\left(\frac{V_{2}^{2}}{V_{1}}\right)\left(e^{-2 \alpha x}-e^{-\alpha x}\right) .
$$

From which we conclude that there exists just one effective potential parameter, which implies into a symmetry of the parameters, leading to the generation of a class of systems with different potential parameters but with the same spectrum.

We could proceed further by discussing many systems presented in the references below, with more or less details. Notwithstanding, we think that the essential feature present in these potentials, as was illustrated through the cases studied here, is that these so called deformed potentials in fact pertain to the same class of potentials as the nondeformed ones. They rather indicate a symmetry of the system where different potential parameters correspond to the same spectrum and do not lead to new physical insights.

Acknowledgments: The author is grateful to $\mathrm{CNPq}$ for partial financial support. 


\section{References}

[1] A. Arai, J. Math. Anal. Appl. 158 (1991) 63; J. Phys. A 34 (2001) 4281.

[2] H. Eğrifes, D. Demirhan and F. Büyükiliç, Phys. Scripta 59 (1999) 90.

[3] H. Eğrifes, D. Demirhan and F. Büyükkiliç, Phys. Scripta 60 (1999) 195.

[4] H. Eğrifes, D. Demirhan and F. Büyükkiliç, Phys. Lett. A 275 (2000) 229.

[5] C. S. Jia, Y. Sun and Y. Li, Phys. Lett. A 305 (2002) 231.

[6] C. S. Jia, P. Y. Lin and L. T. Sun, Physics Letters A 298 (2002) 78.

[7] C. S. Jia, X. L. Zeng, S. C. Li, L. T. Sun and Q. B. Yang, Comm. Theor. Phys. 37 (2002) 523.

[8] C. S. Jia, S. C. Li and L. T. Sun, Phys. Lett. A 300 (2002) 115.

[9] C. S. Jia, X. L. Zeng and L. T. Sun, Phys. Lett. A 294 (2002) 185.

[10] X. Q. Liu and S. Jiang, Physics Letters A 298 (2002) 253.

[11] O. Yelsitas, M. Simsek, R. Server and C. Tezcan, Phys. Scripta 67 (2003) 472.

[12] C. S. Jia, Y. Li, Y. Sun, J. Y. Liu and L. T. Sun, Phys. Lett. A 311 (2003) 115.

[13] C. S. Jia, L. Z. Yi, Y. Sun, J. Y. Liu and L. T. Sun, Mod. Phys. Lett. A 18 (2003) 1247.

[14] G. Chen, Mod. Phys. Lett. A 19 (2004) 2009.

[15] G. Chen, Phys. Scripta 69 (2004) 257.

[16] Y. F. Diao, L. Z. Yi and C. S. Jia, Phys. Lett. A 332 (2004) 157.

[17] C. S. Jia, Y. F. Diao, M. Li, Q. B. Yang, L. T. Sun and R. Y. Huang, J. Phys. A 37 (2004) 11275. 
[18] L. Z. Yi, Y. F. Diao, J. Y. Liu and C. S. Jia, Phys. Lett. A 333 (2004) 212.

[19] M. Simsek and H. Eğrifes, J. Phys. A 37 (2004) 4379.

[20] N. Rosen and P. M. Morse, Phys. Rev. 42 (1932) 210. 